\title{
4D in vivo imaging of glomerular barrier function in a zebrafish podocyte injury model
}

\author{
F. Siegerist,' W. Zhou, ${ }^{2}$ K. Endlich' and N. Endlich' \\ I Department of Anatomy and Cell Biology, University Medicine Greifswald, Greifswald, Germany \\ 2 Department of Pediatrics and Communicable Diseases, University of Michigan, Ann Arbor, MI, USA
}

Received I June 20 I6,
revision requested 27 June 20 I6,
revision received I July 2016,
accepted I0 July 2016
Correspondence: N. Endlich,
Friedrich-Loefflerstr, 23c,
Greifswald I7487, Germany.
E-mail:
nicole.endlich@uni-greifswald.de

See Editorial Commentary: S. Bachmann. 2016. From fish to nephrology: modelling glomerular function in Danio rerio larvae. Acta Physiol (Oxf) 220, 16-18.

\begin{abstract}
Aim: Zebrafish larvae with their simplified pronephros are an ideal model to study glomerular physiology. Although several groups use zebrafish larvae to assess glomerular barrier function, temporary or slight changes are still difficult to measure. The aim of this study was to investigate the potential of in vivo two-photon microscopy (2-PM) for long-term imaging of glomerular barrier function in zebrafish larvae.

Methods: As a proof of principle, we adapted the nitroreductase/metronidazole model of targeted podocyte ablation for 2-PM. Combination with a strain, which expresses eGFP-vitamin D-binding protein in the blood plasma, led to a strain that allowed induction of podocyte injury with parallel assessment of glomerular barrier function. We used four-dimensional (4D) 2-PM to assess eGFP fluorescence over $26 \mathrm{~h}$ in the vasculature and in tubules of multiple zebrafish larvae (5 days post-fertilization) simultaneously.

Results: By 4D 2-PM, we observed that, under physiological conditions, eGFP fluorescence was retained in the vasculature and rarely detected in proximal tubule cells. Application of metronidazole induced podocyte injury and cell death as shown by TUNEL staining. Induction of podocyte injury resulted in a dramatic decrease of eGFP fluorescence in the vasculature over time (about $50 \%$ and $90 \%$ after 2 and $12 \mathrm{~h}$ respectively). Loss of vascular eGFP fluorescence was paralleled by an endocytosis-mediated accumulation of eGFP fluorescence in proximal tubule cells, indicating proteinuria.

Conclusion: We established a microscopy-based method to monitor the dynamics of glomerular barrier function during induction of podocyte injury in multiple zebrafish larvae simultaneously over $26 \mathrm{~h}$.

Keywords glomerular filtration, podocyte injury, proteinuria, proximal tubule.
\end{abstract}

The glomerular filtration barrier of the kidney is composed of three components: the fenestrated endothelial cells, the glomerular basement membrane (GBM) and the podocytes with a slit diaphragm. Podocytes are highly specialized cells with a complex 3D morphology, which is responsible for proper blood filtration in the kidney. These cells cover the outer aspect of the glomerular capillaries by their highly branched cell extensions, the foot processes and interdigitate in a zipper-like fashion with a slit diaphragm in between (Pavenstädt et al. 2003). Impairment of the filtration barrier leads to disruption of the size selectivity and proteinuria, a clinical hallmark of chronic kidney disease. To understand pathogenesis and to screen for 
potential therapies in animal models, which mimic human kidney diseases, quantification of glomerular filtration and proteinuria is undoubtedly important.

The pronephros, the first filtering kidney in developing zebrafish, is an ideal model to study kidney function with a similar glomerular morphology to that of mammals (Drummond \& Davidson 2010). It consists of a single glomerulus connected to a pair of tubules, which starts filtration at 2 days post-fertilization (dpf) and develops a fully working filtration barrier at 3.5 dpf (Drummond 2005, Kramer-Zucker et al. 2005, Drummond \& Davidson 2010).

Several groups used the zebrafish as a model to study defects of the glomerular filtration barrier (Hentschel et al. 2007, Rider et al. 2012, Kotb et al. 2014, Hanke et al. 2015, Wan et al. 2015). Currently, there are two methods to investigate proteinuria in zebrafish. The first one is to compare intravascular fluorescence intensities of either dextran-injected or transgenic zebrafish larvae by fluorescence microscopy. The second approach is to check for tubular endocytosis in histological sections subsequent to intravenous injection of fluorescently labelled $10-$ and $500-\mathrm{kDa}$ dextrans. A disadvantage of both methods is that temporary changes in glomerular barrier function are difficult to measure. Until today, there is no existing method for continuous monitoring of glomerular barrier function in zebrafish larvae over hours or days.

While earlier studies required labour-intensive intravenous injection of fluorescence-labelled molecules such as inulin or dextran for assessment of glomerular filtration, recently, a new transgenic zebrafish strain was established, which endogenously expresses eGFPlabelled vitamin D-binding protein (eGFP-DBP) (Ashworth et al. 2010, Xie et al. 2010, Rider et al. 2012, Kotb et al. 2014). EGFP-DBP $(78 \mathrm{kDa})$, a protein of the albumin family, is synthetized in the liver under control of the liver-type fatty acid-binding protein (1-fabp) promotor and secreted into the blood plasma. Under physiological conditions, eGFP-DBP is retained in the vasculature due to the size selectivity of the glomerular filtration barrier. After impairment of the filtration barrier, a decrease of the intravascular eGFP-DBP and megalin-mediated endocytosis of eGFP-DBP in proximal tubule cells (PTCs) were described (Ashworth et al. 2010, Kotb et al. 2014, 2016, Wan et al. 2015).

Due to the availability of transparent and fluorophore-expressing strains, zebrafish larvae are a powerful model for microscopy-based in vivo analysis of the glomerular filtration barrier, especially by longterm two-photon microscopy (2-PM) (Endlich et al. 2014, Kotb et al. 2016). To study the development and dynamics of proteinuria in zebrafish larvae in vivo, we used the nitroreductase (NTR)/metronidazole (MTZ) model of targeted podocyte ablation. This zebrafish strain expresses the Escherichia coli-derived enzyme NTR under the control of the podocyte-specific podocin (nphs2) promotor. After application of the prodrug MTZ to the medium of the larvae, podocytes become injured, apoptotic and detach (Zhou \& Hildebrandt 2012, Huang et al. 2013). In this study, we utilized different zebrafish strains to generate a new transgenic zebrafish strain that enables long-term in vivo 2-PM imaging to induce and track changes of glomerular barrier function over up to $26 \mathrm{~h}$.

\section{Materials and methods}

\section{Zebrafish stocks}

Zebrafish stocks and larvae were maintained as described previously (Müller et al. 2011, Kotb et al. 2014). The Cade strain ( $\operatorname{Tg}\left(l\right.$-fabp:eGFP-DBP); mitfa $a^{w 2 /}$ ${ }^{w 2}$; roy ${ }^{a 9 / a 9}$ ) expresses the $78-\mathrm{kDa}$ eGFP-vitamin Dbinding protein in the transparent Casper background (Xie et al. 2010, Kotb et al. 2014). The $\operatorname{Tg}(n p h s 2$ : Eco.NfsB-mCherry) strain (Zhou \& Hildebrandt 2012) was cross-bred with Casper (mitfa ${ }^{w 2 / w 2}$; roy ${ }^{a 9 / a 9}$ ) (White et al. 2008) resulting in a new strain named Nury (Tg(nphs2:Eco.NfsB-mCherry); mitfa $a^{w 2 / w 2}$; roy $\left.{ }^{a 9 / a 9}\right)$. For evaluation of the glomerular barrier function, Nury was cross-bred with Cade resulting in a new transparent strain with eGFP-DBP in the blood plasma and NTR-mCherry in podocytes ( $\mathrm{Tg}$ (l-fabp: eGFP-DBP); $\operatorname{Tg}$ (nphs2:Eco.NfsB-mCherry) mitfa $a^{w 2 / w 2}$; roy $\left.{ }^{a 9 / a 9}\right)$. This strain was named BlooP for blood and podocytes. All experiments were performed in accordance with German law and were overseen by the agencies of the federal state of Mecklenburg-Western Pomerania. All 2-PM experiments were performed at $22{ }^{\circ} \mathrm{C}$. Metronidazole (Sigma-Aldrich, St. Louis, MO, USA) was freshly prepared in $0.1 \%$ DMSO-E3. Zebrafish larvae were treated at 3 and $5 \mathrm{dpf}$ with 1 or $5 \mathrm{~mm} \mathrm{MTZ.}$

\section{Histology}

Cryosections and confocal microscopy were performed as described elsewhere (Endlich et al. 2014). TUNEL assay (in situ Cell Death Detection Kit, Fluorescein, Roche, Basel, Switzerland) was prepared according to the manufacturer's description followed by $0.013 \mathrm{mg} \mathrm{mL}^{-1}$ Hoechst 33342 (Sigma-Aldrich) and mounting in Mowiol (Carl Roth, Karlsruhe, Germany).

\section{Imaging}

For in vivo imaging, up to ten larvae at $5 \mathrm{dpf}$ were embedded as described before (Endlich et al. 2014). After hardening, the larvae were covered with $1 \mathrm{~mm}$ 
Figure I (a) Positive TUNEL signal in cross sections of Nury larvae after 20-h exposition to $5 \mathrm{~mm}$ MTZ. In contrast to control larvae, a decrease of mCherry fluorescence of podocytes was found in Nury larvae $(n=3$ individual experiments, scale bar represents $10 \mu \mathrm{m})(\mathrm{b})$ TUNEL signal colocalized with remaining mCherry fluorescence and nuclear Hoechst staining (scar bar represents $5 \mu \mathrm{m})$. (a)
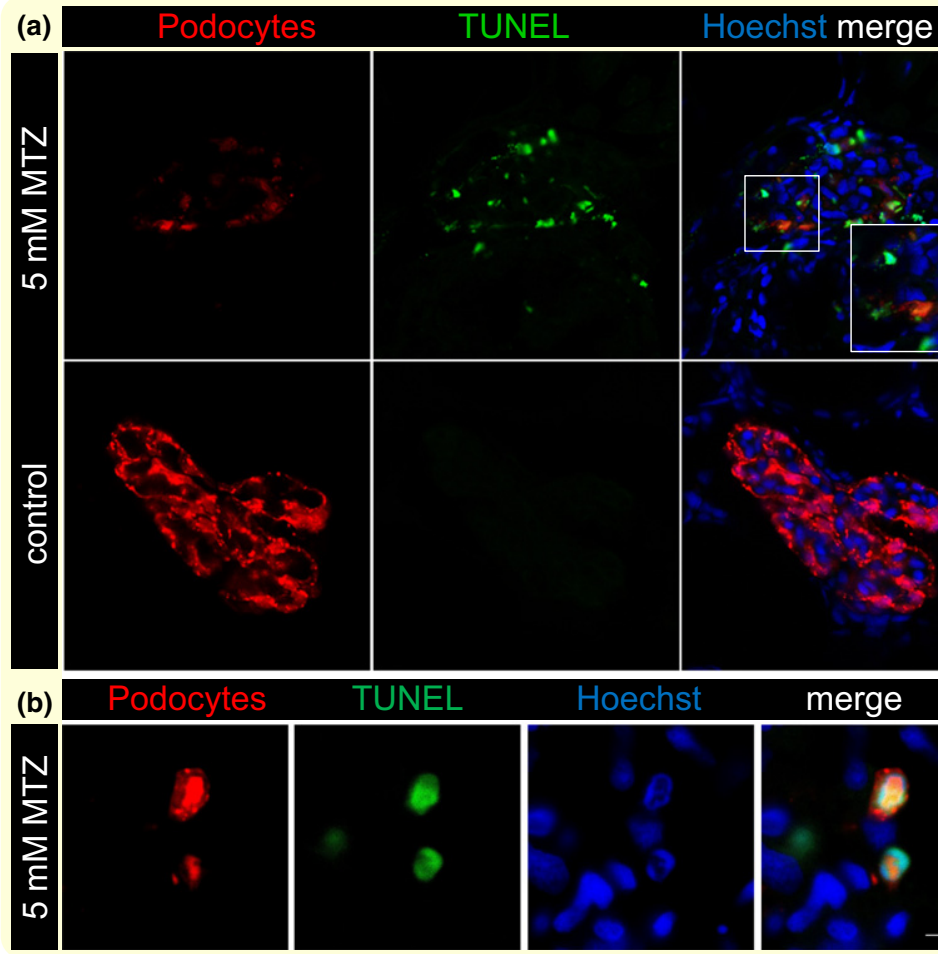

Hoechst merge

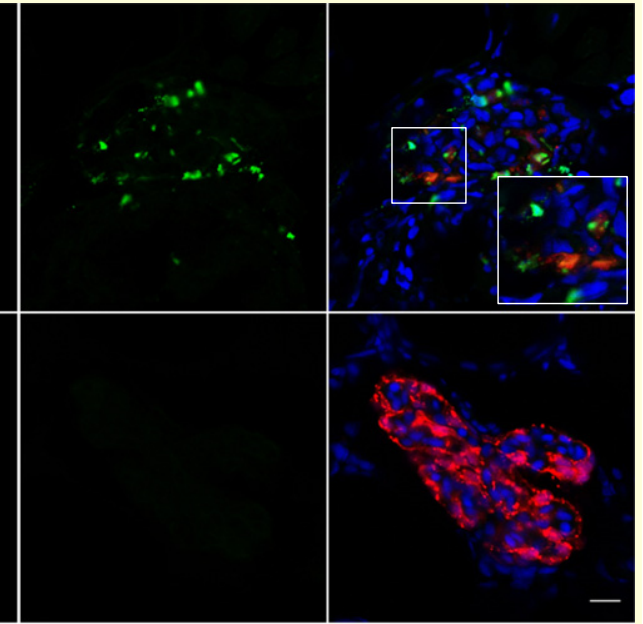

TUNEL

merge
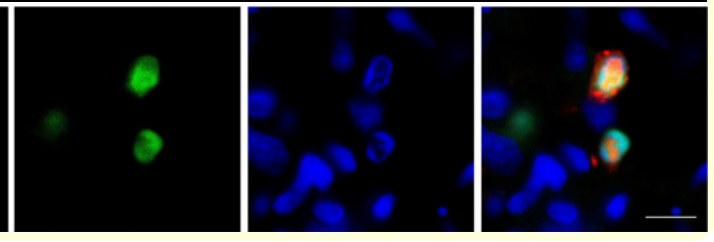

MTZ in $0.1 \%$ DMSO in E3 medium containing $0.1 \%$ tricaine. 2-PM was performed with a LSM710MP (Carl Zeiss Microimaging, Jena, Germany) and $20 \times$ (1.0 NA) water immersion objective with a pulsed Ti-sapphire laser (Chamaeleon, Coherent, Santa Clara, CA, USA). In every $30 \mathrm{~min}$, automated $z$-stacks of each larva over $118 \mu \mathrm{m}$ were recorded. Fluorescence measurements and 3D reconstruction were performed with Zeiss ZEN 2010 software (Carl Zeiss Microimaging) and arranged to $4 \mathrm{D}$ movies with IMAGEJ (National Institutes of Health, Bethesda, MD, USA). Statistics (Student's $t$-test) were calculated with EXCEL (version 14.0.7, Microsoft, Redmond, WA, USA).

\section{Results}

Application of MTZ to Nury larvae promotes apoptosis in podocytes

We crossed the $\operatorname{Tg}(n p h s 2$ :Eco.NfsB-mCherry) zebrafish strain, which expresses the prokaryotic enzyme NTR and mCherry under the control of the podocytespecific podocin promoter (Zhou \& Hildebrandt 2012) with the transparent zebrafish Casper (mitfa ${ }^{w 2 / w 2}$; roy $^{\text {a9/a9 }}$ ) (White et al. 2008, Zhou \& Hildebrandt 2012). In order to induce proteinuria, larvae (3 dpf) of the new strain (Nury - nitroreductase-mCherry) were incubated for $20 \mathrm{~h}$ with $5 \mathrm{~mm}$ MTZ. To verify MTZ-induced apoptosis in Nury larvae, sections were stained by terminal deoxynucleotidyl transferase dUTP nick-end labelling (TUNEL). In contrast to DMSO $(0.1 \%)$-treated larvae (control), a widespread TUNEL signal was detected (Fig. 1a), which colocalized with podocytes and Hoechst-labelled nuclei (Fig. 1b), indicating specific cell death of podocytes.

\section{Live assessment of glomerular barrier function during podocyte injury}

For further assessment of the glomerular barrier function, we established a new zebrafish strain (BlooP) on the transparent Casper background, which additionally expresses the 78-kDa eGFP-labelled Vitamin D-binding protein (eGFP-DBP) under control of the liver-type fatty acid-binding protein promotor in the blood plasma.

To verify expression of NTR-mCherry at $5 \mathrm{dpf}$, we performed 2-PM two-channel scans for mCherry and eGFP (Fig. 2a). The following scans captured eGFP fluorescence alone to minimize bleaching effects by the significantly shorter excitation wavelength of mCherry (Fig. 2b-f). To induce proteinuria, $1 \mathrm{~mm}$ MTZ was added to the medium of BlooP larvae at $t=0$. MTZ treatment led to a significant decrease of relative eGFP fluorescence intensity in the dorsal aorta of BlooP $(n=33)$ as compared to Cade control larvae $(n=28)$. At $t=24 \mathrm{~h}$, a decrease to $0.6 \%$ was measured $(P<0.001$, Student's $t$-test, Fig. $2 \mathrm{~g})$.

Additionally, a significant increase of the eGFP fluorescence in PTCs was detected beginning at $t=12 \mathrm{~h}$ due to an endocytic uptake of eGFP-DBP, which 


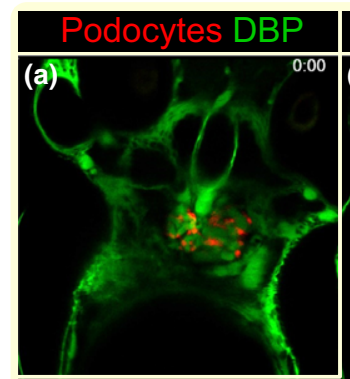

(d)

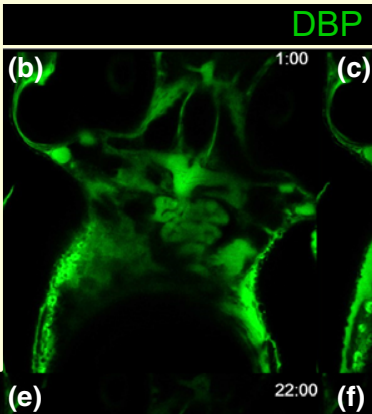

(c)
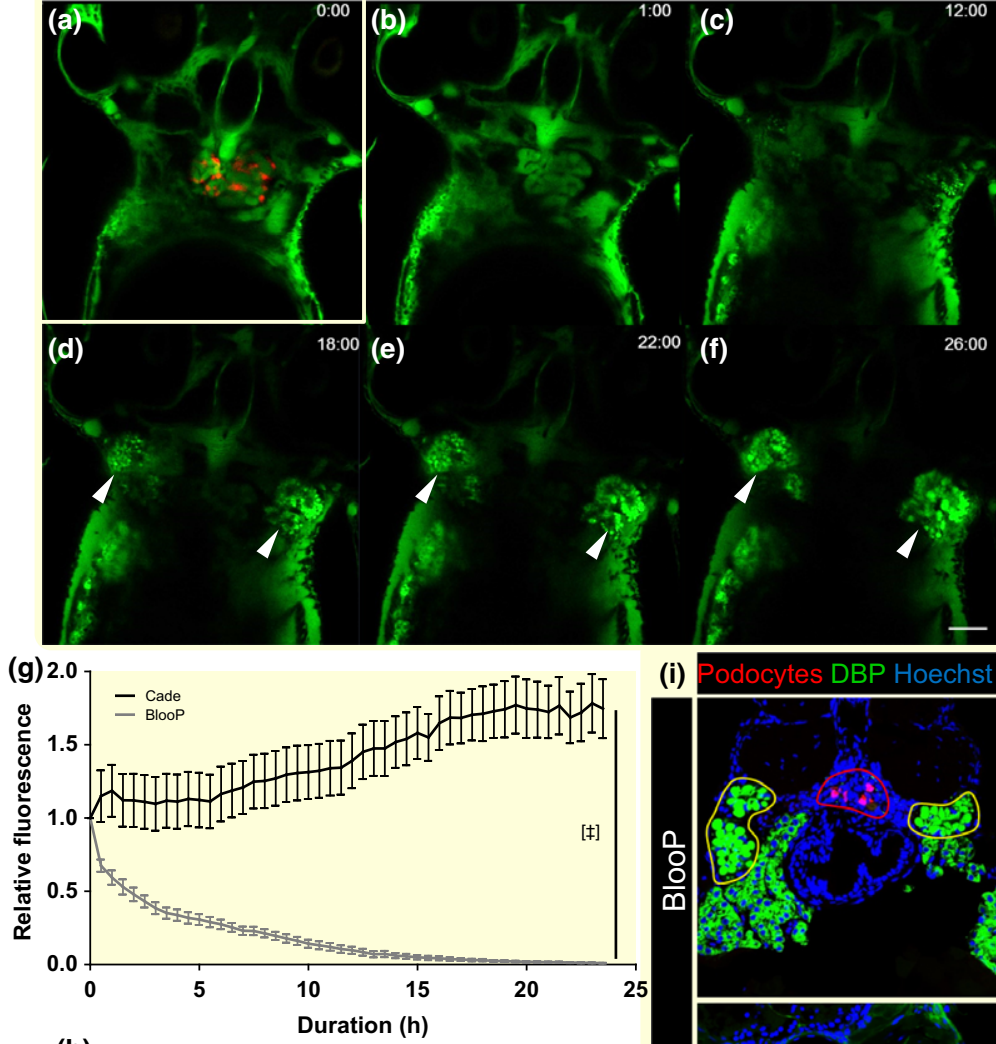

(h)

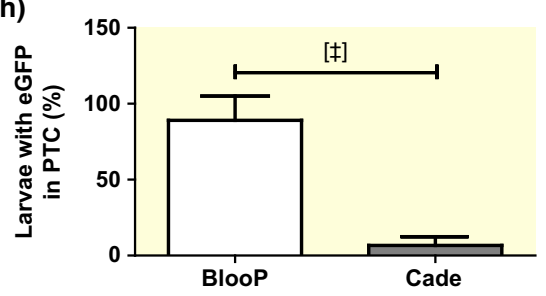

(i)
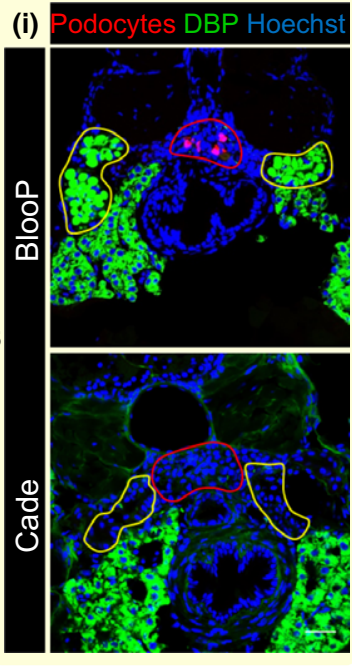

Figure 2 Single frames from $\mathrm{z}$-stacks of a living BlooP larva exposed to $1 \mathrm{~mm}$ MTZ over 26 h. mCherry and eGFP multi-track scan at $t=0: 00$ (a). Following eGFP scans up to $t=26: 00 \mathrm{~h}$ show progressive accumulation of eGFP-DBP in PTCs (arrowheads) and a decrease of fluorescence in vasculature $(\mathrm{b}-\mathrm{f})$. Scale bar represents $50 \mu \mathrm{m}$. BlooP larvae showed a significant higher decrease of the relative eGFP fluorescence intensity compared to control (Cade) measured in the dorsal aorta $(\mathrm{g})$. Mean values of $n=30 \mathrm{BlooP}$ larvae from three individual experiments compared to $n=28$

Cade larvae. Error bars indicate standard error mean. [\$]: $P<0.001$. (h) shows that $89 \%(\mathrm{SD}=13.9 \%, n=33)$ of BlooP larvae treated with $1 \mathrm{~mm} \mathrm{MTZ}$ showed accumulation of eGFP in PTCs compared to $6.7 \%$ of control larva $(\mathrm{SD}=4.6 \%, n=28)$. Only eGFP-DBP accumulation was observed by confocal microscopy of cryosections in larvae that showed appearance of eGFP-DBP in 2PM (i). The red line in picture I encircles the position of the glomerulus and the yellow line of the proximal tubule. Ventral eGFP signal is due to $l$-fabp expression in hepatocytes (representative images of $n=3$ independent experiments; scale bar represents $25 \mu \mathrm{m}$ ). passed the leaky filtration barrier after podocyte injury (Fig. 2c-f; movie S1). This increase was found in $89 \%$ $(\mathrm{SD}=13.9 \%, P=0.0002)$ of BlooP larvae $(n=33)$ compared to $6.7 \%(\mathrm{SD}=4.6 \%)$ of control larvae $(n=28)$ in three individual experiments (Fig. 2h).

While Figure $2 \mathrm{i}$ shows an intensive eGFP signal in PTCs (typical position of PTCs is marked by yellow circles) in a cross section of a BlooP larva (6 dpf) after 2PM, PTCs of the shown Cade control larva show no accumulation of eGFP-DBP. However, only larvae (BlooP or Cade) showing eGFP-DBP accumulation in 2-PM showed eGFP-positive PTCs in confocal microscopy of cryosections (in $n=3$ individual experiments).

\section{$4 D$ in vivo imaging of proteinuria}

Movie S2 offers a unique 4D view on the particular changes following podocyte injury. At $t=0$, the glomerular tuft is visible ventral to the dorsal aorta (Fig. 3 arrows). EGFP appears between $t=12 \mathrm{~h}$ and $t=13 \mathrm{~h}$ in PTCs and unveils its contorted 3D structure. Over the whole period of MTZ treatment, the intravascular fluorescence intensity of BlooP larvae decreases due to the loss of eGFP-DBP from the blood as shown in movie S3. In contrast to that, the intravascular fluorescence intensity of Cade larvae increases over the imaging period.

\section{Discussion}

Here, we demonstrate that the zebrafish larva is an ideal model to study the glomerular filtration barrier and the development of proteinuria in vivo.

For the last decades, the most popular model organisms in renal research have been rodents. Despite of their many advantages, mice and rats lack easy accessibility of glomeruli and only strains with superficial glomeruli, like Munich-Wistar rats, are suitable for in vivo imaging (Russo et al. 2007, Schießl et al. 2016). Additionally, most in vivo multi photon- 
Figure 3 Single frames of 3D reconstructions of z-stacks of BlooP and Cade larvae show enhanced spatial discrimination of the progress of proteinuria induced by MTZ treatment while Cade control larvae showed increasing intravascular fluorescence (arrows: glomerular tuft, asterisks: dorsal aorta, arrowheads: PTCs; scale bar represents $50 \mu \mathrm{m})$.

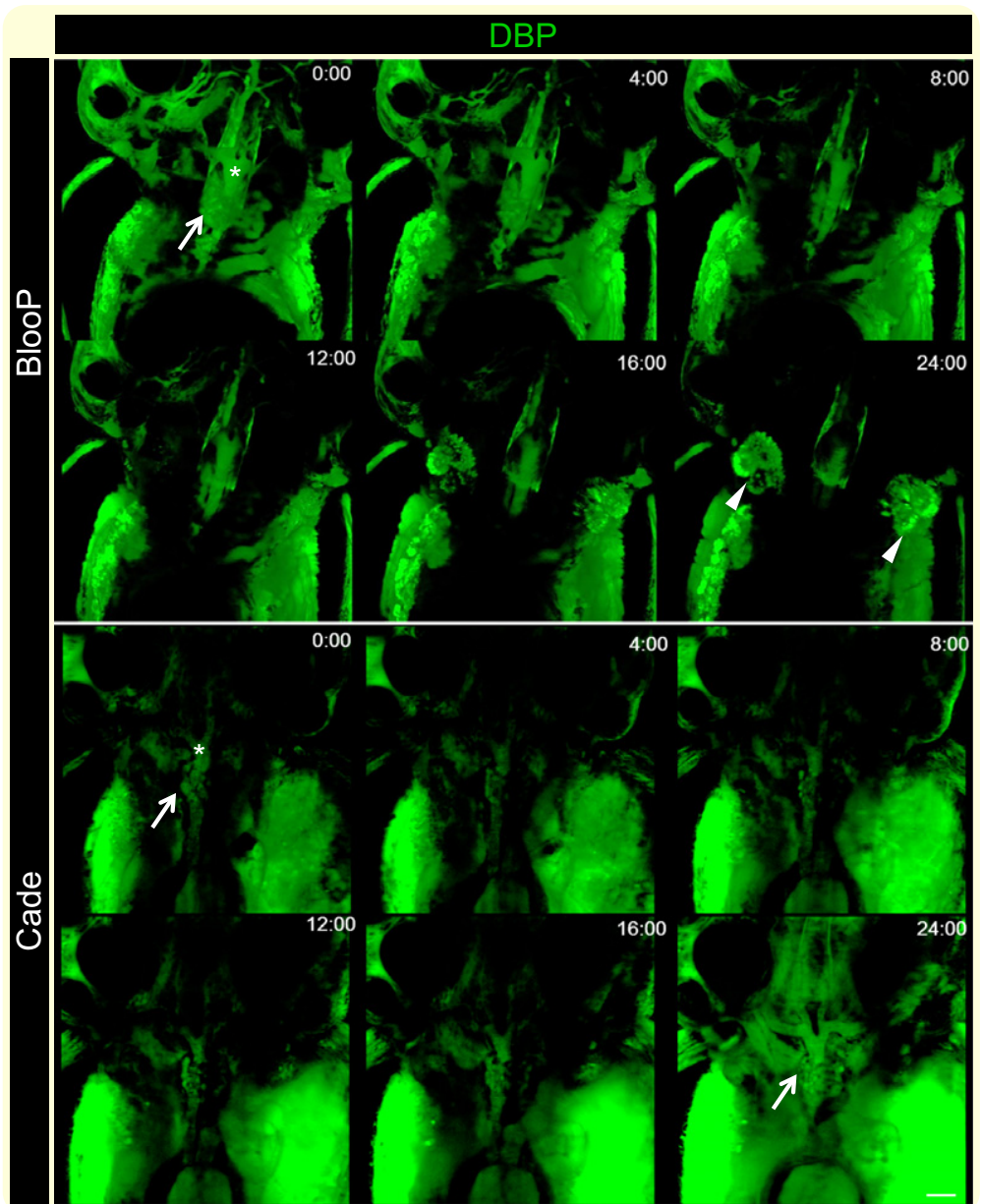

imaging approaches in mammals require rather complex and time-consuming preparations until filtering glomeruli can be visualized (Peti-Peterdi \& Sipos 2010, Brahler et al. 2016, Schießl et al. 2016) or appear to be rather artificial (Kistler et al. 2014). In contrast to that, our model has no limitations in accessibility of glomeruli and can be applied to all available zebrafish strains, mutants, and especially gene knockdown strategies for evaluation of specific protein function, for example with morpholinos. Moreover, due to semi-automated simultaneous imaging of a group of larvae, our technique offers the opportunity for an increased throughput analysis of differently treated zebrafish larvae to evaluate the potential of particular treatments prior to labour- and cost-intensive experiments in a higher vertebrate model.

Although there are broad similarities between the larval zebrafish and mammalian glomerular filtration, there are also important differences. Compared to mammals, the arterial blood pressure reaches a relatively low maximum systolic pressure of about $0.5 \mathrm{mmHg}$ at $4 \mathrm{dpf}$ (Pelster \& Burggren 1996).
Additionally, the major part of tubular flow in larval zebrafish is dependent on the function of motile cilia in the pronephros (Kramer-Zucker et al. 2005). Like most teleost species, zebrafish lack the albumin gene but possess another similar protein of the albumin family, the vitamin D-binding protein gene $(d b p)$ (Noel et al. 2010), which was labelled by eGFP in the Cade strain. As both proteins have a similar size (66$\mathrm{kDa}$ albumin vs. $78-\mathrm{kDa}$ eGFP-DBP), it is possible to study the glomerular barrier function in real time with this transgenic strain.

Compared to BlooP larvae treated with $1 \mathrm{~mm}$ MTZ, the control larvae (Cade) showed increasing expression of eGFP-DBP as seen by eGFP fluorescence in the vasculature during the imaging period between 5 and $6 \mathrm{dpf}$. These findings are consistent with previous investigations that showed increasing activity of the l-fabp promotor between 4 and $7 \mathrm{dpf}$ in larval zebrafish (Her et al. 2003, 2004).

In this study, we underline the important role of podocytes for the integrity of the intact glomerular filtration barrier. In contrast to Russo and co-workers who postulated that the filtration barrier leaks 
protein, especially albumin, at nephrotic levels, which is salvaged by PTCs (Russo et al. 2007), we only observed accumulation of eGFP-DBP in PTCs under healthy conditions in $6.7 \%$ of Cade control larvae, indicating that loss of eGFP-DBP from the vasculature of healthy zebrafish larvae is a rather rare event. However, after impairment of the filtration barrier through MTZ-induced podocyte injury (Pisharath et al. 2007, Zhou \& Hildebrandt 2012), we found a rapid increase of endocytic uptake of filtered eGFPDBP in PTCs.

Previous studies which investigated glomerular filtration with eGFP-labelled DBP in zebrafish focused on the measurement of fluorescence intensity in the eye (Hanke et al. 2015) or in the vasculature (Kotb et al. 2014). Another approach is to measure levels of filtered eGFP-DBP in the medium of a group of zebrafish larvae by dot blot or by ELISA analysis for eGFP (Zhou \& Hildebrandt 2012, Hanke et al. 2015). A disadvantage of the two latter methods is that temporary and intermittent alterations of the glomerular filtration barrier are hardly detectable. We have overcome this disadvantage by continuous measurement of eGFP fluorescence over $26 \mathrm{~h}$ so that even temporary changes of the eGFP-DBP fluorescence are detectable. Faster (within minutes) alterations of the intravascular fluorescence intensity can also be tracked with our technique, simply using a shorter recording interval.

Taken together, this animal model of the glomerular filtration barrier allows us to follow the development and dynamics of proteinuria in 4D in a group of larvae over extended time periods of $26 \mathrm{~h}$ in vivo.

\section{Conflicts of interest}

None.

The excellent technical assistance of Oliver Zabel is greatly acknowledged. This study was supported by a grant of the European Union within the 7th framework program to K.E. (project 'EnVision', grant agreement no. 264143), by grants of the German Federal Ministry of Education and Research (BMBF) to N.E. (project 'STOP-FSGS', grant no. 01GM1518B) and was supported by a scholarship of the Gerhard Domagk program of the University Medicine Greifswald to F.S.

\section{References}

Ashworth, S., Teng, B., Kaufeld, J., Miller, E., Tossidou, I., Englert, C., Bollig, F., Staggs, L., Roberts, I.S.D., Park, J.-K., Haller, H. \& Schiffer, M. 2010. Cofilin-1 inactivation leads to proteinuria-studies in zebrafish, mice and humans. PLoS One 5, e12626.

Brahler, S., Yu, H., Suleiman, H., Krishnan, G.M., Saunders, B.T., Kopp, J.B., Miner, J.H., Zinselmeyer, B.H. \& Shaw, A.S.
2016. Intravital and kidney slice imaging of podocyte membrane dynamics. J Am Soc Nephrol [Epub ahead of print].

Drummond, I.A. 2005. Kidney development and disease in the zebrafish. J Am Soc Nephrol 16, 299-304.

Drummond, I.A. \& Davidson, A.J. 2010. Zebrafish kidney development. Methods Cell Biol 100, 233-260.

Endlich, N., Simon, O., Göpferich, A., Wegner, H., Moeller, M.J., Rumpel, E., Kotb, A.M. \& Endlich, K. 2014. Twophoton microscopy reveals stationary podocytes in living zebrafish larvae. J Am Soc Nephrol 25, 681-686.

Hanke, N., King, B.L., Vaske, B., Haller, H. \& Schiffer, M. 2015. A fluorescence-based assay for proteinuria screening in larval zebrafish (Danio rerio). Zebrafish 12, 372-376.

Hentschel, D.M., Mengel, M., Boehme, L., Liebsch, F., Albertin, C., Bonventre, J.V., Haller, H. \& Schiffer, M. 2007. Rapid screening of glomerular slit diaphragm integrity in larval zebrafish. Am J Physiol Renal Physiol 293, F1746-F1750.

Her, G.M., Chiang, C.C., Chen, W.Y. \& Wu, J.L. 2003. In vivo studies of liver-type fatty acid binding protein (L-FABP) gene expression in liver of transgenic zebrafish (Danio rerio). FEBS Lett 538, 125-133.

Her, G.M., Chiang, C.C. \& Wu, J.L. 2004. Zebrafish intestinal fatty acid binding protein (I-FABP) gene promoter drives gut-specific expression in stable transgenic fish. Genesis 38, 26-31.

Huang, J., McKee, M., Huang, H.D., Xiang, A., Davidson, A.J. \& Lu, H.A.J. 2013. A zebrafish model of conditional targeted podocyte ablation and regeneration. Kidney Int 83, 1193-1200.

Kistler, A.D., Caicedo, A., Abdulreda, M.H., Faul, C., Kerjaschki, D., Berggren, P.O., Reiser, J. \& Fornoni, A. 2014. In vivo imaging of kidney glomeruli transplanted into the anterior chamber of the mouse eye. Sci Rep 4, 3872.

Kotb, A.M., Müller, T., Xie, J., Anand-Apte, B., Endlich, K. \& Endlich, N. 2014. Simultaneous assessment of glomerular filtration and barrier function in live zebrafish. Am J Physiol Renal Physiol 307, 34.

Kotb, A.M., Simon, O., Blumenthal, A., Vogelgesang, S., Dombrowski, F., Amann, K., Zimmermann, U., Endlich, K. \& Endlich, N. 2016. Knockdown of ApoL1 in zebrafish larvae affects the glomerular filtration barrier and the expression of nephrin. PLoS One 11, e0153768.

Kramer-Zucker, A.G., Wiessner, S., Jensen, A.M. \& Drummond, I.A. 2005. Organization of the pronephric filtration apparatus in zebrafish requires Nephrin, Podocin and the FERM domain protein Mosaic eyes. Dev Biol 285, 316329.

Müller, T., Rumpel, E., Hradetzky, S., Bollig, F., Wegner, H., Blumenthal, A., Greinacher, A., Endlich, K. \& Endlich, N. 2011. Non-muscle myosin IIA is required for the development of the zebrafish glomerulus. Kidney Int 80, 1055 1063.

Noel, E.S., Reis, M.D., Arain, Z. \& Ober, E.A. 2010. Analysis of the Albumin/alpha-Fetoprotein/Afamin/Group specific component gene family in the context of zebrafish liver differentiation. Gene Expr Patterns 10, 237-243.

Pavenstädt, H., Kriz, W. \& Kretzler, M. 2003. Cell biology of the glomerular podocyte. Physiol Rev 83, 253-307. 
Pelster, B. \& Burggren, W.W. 1996. Disruption of hemoglobin oxygen transport does not impact oxygen-dependent physiological processes in developing embryos of zebra fish (Danio rerio). Circ Res 79, 358-362.

Peti-Peterdi, J. \& Sipos, A. 2010. A high-powered view of the filtration barrier. J Am Soc Nephrol 21, 1835-1841.

Pisharath, H., Rhee, J.M., Swanson, M.A., Leach, S.D. \& Parsons, M.J. 2007. Targeted ablation of beta cells in the embryonic zebrafish pancreas using E. coli nitroreductase. Mech Dev 124, 218-229.

Rider, S.A., Tucker, C.S., del-Pozo, J., Rose, K.N., MacRae, C.A., Bailey, M.A. \& Mullins, J.J. 2012. Techniques for the in vivo assessment of cardio-renal function in zebrafish (Danio rerio) larvae. J Physiol 590, 1803-1809.

Russo, L.M., Sandoval, R.M., McKee, M., Osicka, T.M., Collins, A.B., Brown, D., Molitoris, B.A. \& Comper, W.D. 2007. The normal kidney filters nephrotic levels of albumin retrieved by proximal tubule cells. Retrieval is disrupted in nephrotic states. Kidney Int 71, 504-513.

Schießl, I.M., Hammer, A., Kattler, V., Gess, B., Theilig, F., Witzgall, R. \& Castrop, H. 2016. Intravital imaging reveals angiotensin II-induced transcytosis of albumin by podocytes. J Am Soc Nephrol 27, 731-744.

Wan, X., Chen, Z., Choi, W.-I., Gee, H.Y., Hildebrandt, F. \& Zhou, W. 2015. Loss of epithelial membrane protein 2 aggravates podocyte injury via upregulation of caveolin-1. J Am Soc Nephrol 27, 1066-1075.

White, R.M., Sessa, A., Burke, C., Bowman, T., LeBlanc, J., Ceol, C., Bourque, C., Dovey, M., Goessling, W., Burns, C.E. \& Zon, L.I. 2008. Transparent adult zebrafish as a tool for in vivo transplantation analysis. Cell Stem Cell 2, 183-189.

Xie, J., Farage, E., Sugimoto, M. \& Anand-Apte, B. 2010. A novel transgenic zebrafish model for blood-brain and blood-retinal barrier development. BMC Dev Biol 10, 76.

Zhou, W. \& Hildebrandt, F. 2012. Inducible podocyte injury and proteinuria in transgenic zebrafish. J Am Soc Nephrol 23, 1039-1047.

\section{Supporting Information}

Additional Supporting Information may be found online in the supporting information tab for this article:

Movie S1. Movie S1 shows 2-PM z-stacks of a BlooP larva at $5 \mathrm{dpf}$ at $t=1,7.5,12.5,26 \mathrm{~h}$ in $1 \mathrm{~mm}$ MTZ.

Movie S2. Movie S2 shows a 4D reconstruction of a BlooP larva at $5 \mathrm{dpf}$ over $26 \mathrm{~h}$ in $1 \mathrm{~mm}$ MTZ. The decrease of eGFP fluorescence in the blood and accumulation of eGFP-DBP in the PTCs can be distinguished over the time.

Movie S3. Movie S3 shows the 4D reconstruction of a Cade larva at 5 to $6 \mathrm{dpf}$ and serves as control to movie S2. An increase of the fluorescence intensity in the blood can be seen as well as no accumulation of eGFP in PTCs compared to movie S2. 